\title{
A Synthesized Evaluation on Supply Chain Risk Based on Variable-weights Extenics Matter-Element Model
}

\author{
Zhong Changbao ${ }^{1,2}$,Wei Xiaoping ${ }^{2}$ \\ [1] School of Business, Huaihai Institute of Technology \\ [2] School of Management, China University of Mining and Technology \\ JiangSu XuZhou 221008; JiangSu Lian Yun-gang 222001 \\ e-mail : zhongchangbao@126.com
}

\begin{abstract}
In view of the supply chain risk evaluation has become the hot-issue and nodus about the supply chain risk management, and it is difficult to embody the requirements about balanced and incentive by the constant weight synthesize in evaluation, then set up a supply chain risk evaluation model based on variable-weights extenics matter-element, by combining the extenics theory with the variable-weights theory; and apply this model to evaluate the risk of Hengrui supply chain, based on the risk evaluation factors in the supply chain system, which supposed by the author. The results of the evaluation shows that the model not only overcomes the drawbacks of constant weights synthesize, motivate some key influenced factors, but also shows that the model has good scientificity, flexibility and effectiveness.
\end{abstract}

Key Words: Supply Chain Risk; Variable-Weights Theory; Extenics; Matter-Element Model

\section{Introduction}

The supply chain is a network chain structure formed in production and circulation process, and involved of upstream and downstream enterprises, its main purpose is to deliver goods or services to its final customers. However, in recent years, the risks such as the economic crisis, natural disasters, network security, increasing competition, random demand and so on, are more and more seriously influence the security and stability about the supply chain, and make the supply chain more and more weakly, and also bring about damage and loss to upstream and downstream enterprises, even the whole supply chain without exception, this make supply chain risk management to be a important subject in supply chain research necessarily, moreover, evaluating the supply chain risk correctly is the most important link in carrying out the supply chain risk management, evaluating the supply chain risk is measuring and evaluating the factors about supply chain risk quantitative, composed of evaluates each factor in supply chain and synthesize evaluates the supply chain risk. However, in general, there are few literatures about supply chain risk evaluation, Prater and some others introduced a scoring method about global supply chain to measure the risks and give the applicable case; in the view of the probability and the result of risk event, Jukka and some others researched supply chain risk evaluation semi-quantitatively; Ding Weidong and some others introduced supply chain reliability evaluation matrix to evaluate the supply chain risk quantitatively; Xiao Meidan and some others introduced supply chain risk evaluation index system and evaluated the supply chain risk based on uncertainty theory and fuzzy method; Zhang Yanru and some others evaluated the supply chain uncertain risks which have different preferences based on fuzzy evaluation method.

All these methods have their own characteristics and advantages, and came from the hard work of the scholars, but there are some inadequacies, for example, some of the methods can not be executed strictly in practice; the evaluation index can not fit some design principles, such as scientificity、 comparability、 operability and so on.; some evaluation methods in calculating the evaluation values are based on the constant-weighs synthesis, and are difficult to reflect the attention to some risk factors in a risk environment about supply chain.

This paper will evaluate the supply chain risks by using extenics based on the existed literatures, that is determining the risk categories of the supply chain risk finally, by using calculating the weight and associated degrees of the risk category about the factors of the supply chain risk. And using the variable-weights theory which introduced by professor Wang Peizhuang, when calculating the weight of the evaluation factors of the supply chain risk(many scholars researched the theory and application about the variable-weights, please refer to references [7-10]), to reflect the attention about some certain risk factors in a risk circumstance by the weight, and improve the scientificity and accuracy of the evaluation result, the author will introduce the system of the supply chain risk evaluation, and give the application of the evaluation method.

\section{Setting up the variable weights extension matter element model}

Extenics is proposed by Cai Wen a Chinese scholars in 1983 ,which is a rule and method that start with the rules of conversion and the solutions of incompatible problems, by introducing matter-elements, then changing and calculating about them, to research and solve the incompatible problems qualitatively and quantitatively, this method take the matter-element theory and extenics as framework, the matter-element as logic cell, and the matter-element is take the ordered triples $R=(N, c, v)$ as the basic element to describe one thing ( $N$ is the name, $C$ is the characteristic, $v$ is the value of the thing, they are also called three elements of the matter-element).

\subsection{Constructing the matrix of the multi-dimensional}

characteristics matter-element

Hypothesizing one thing $N$ has $n$ characteristics, 
each of the characteristics called $c_{1}, c_{2}, \cdots, c_{n}$, and the Corresponding value are $v_{1}, v_{2}, \cdots, v_{n}$, constructing the matrix of the multi-dimensional characteristics matter-element:

$$
R=\left(N, C_{i}, V_{i}\right)=\left[\begin{array}{ccc}
N & c_{1} & v_{1} \\
& c_{2} & v_{2} \\
& \cdots & \cdots \\
& c_{n} & v_{n}
\end{array}\right]
$$

Because the different thing has different characterized matter-element matrix, and the same thing also has different characterized matter-element matrix while not at the same time. As a result, we can use the characteristic matter-element matrix to distinguish the different things, or to know which stage does the thing at.

\subsection{Determining the classical domain and section domain}

Hypothesizing there are $n$ main factor indexes of the supply chain risk evaluation, each of them called $f_{1}, f_{2}, \cdots, f_{n}$, the experts or according to the statistics cluster analysis, dividing the supply chain risk into many categories qualitatively, based on these factor indexes, describing them as the following qualitative or quantitative synthesis evaluated matter-element models, and called classical domain matter-element matrix

$$
R_{j}=\left(N_{j}, F_{i}, V_{j i}\right)=\left[\begin{array}{ccc}
N_{j} & f_{1} & \left\langle a_{j 1}, b_{j 1}\right\rangle \\
& f_{2} & \left\langle a_{j 2}, b_{j 2}\right\rangle \\
\ldots & \ldots \\
& f_{n} & \left\langle a_{j n}, b_{j n}\right\rangle
\end{array}\right]
$$

In the formula, $R_{j}$ stands for the matter-element model when the supply chain risk at $j$ stage, $f_{i}$ stands for the $i$ factor index, ${ }^{N}{ }_{j}$ stands for the $j$ risk category, $V_{j i}$ stands for the value range that determined by the $i$ factor index $f_{i}$ about the ${ }^{j}$ risk category $N_{j}$

$$
R_{p}=\left(N_{p}, F, V_{p}\right)=\left[\begin{array}{ccc}
N_{p} & f_{1} & \left\langle a_{p 1}, b_{p 1}\right\rangle \\
& f_{2} & \left\langle a_{p 2}, b_{p 2}\right\rangle \\
\cdots & \ldots \\
& f_{n} & \left\langle a_{p n}, b_{p n}\right\rangle
\end{array}\right]
$$

In the formula, $p$ stands for the whole of the risk categories, $V_{p i}=\left\langle a_{p i}, b_{p i}\right\rangle$ is the value range of $p$ that can be got from the factor index $f_{i}$.

\subsection{Determining the risk category of the supply chain and the} matter-element which to be evaluated

The grading standards of the supply chain risk category are determined by a group of experts, divided the grades of the supply chain risk category into 5 categories: Low-risk I, the lower risk II, Medium-risk III, the higher risk IV, high-risk $\mathrm{V}$,each of these 5 categories be assigned 1,3,5,7,9. By the same principle, the evaluated factor index can also refer this way, invited some experts to evaluate all Eigen values about a supply chain risk matter-element $R_{t}$ according to the actual case, and score them according to the given standards, then get the matter-element which to be evaluated

$$
R_{t}=\left(T, F_{i}, V_{i}\right)=\left[\begin{array}{ccc}
T & f_{1} & v_{1} \\
& f_{2} & v_{2} \\
& \cdots & \cdots \\
& f_{n} & v_{n}
\end{array}\right]
$$

In the formula, $T$ stands for the risk of the to be evaluated supply chain, $V_{i}$ stands for the value of the $i$ factor index of the supply chain which to be evaluated, that is to say $V_{i}$ is the value about $f_{i}$.

\subsection{Determining the correlation about each risk category of} each evaluated index of the matter-element which to be evaluated

The correlations of each single evaluated factor index about each risk category among the supply chain risk are:

$$
\begin{aligned}
& k_{j}\left(v_{i}\right)= \begin{cases}\frac{-\rho\left(v_{i}, v_{j i}\right)}{\left|v_{j i}\right|} & \mathrm{v}_{i} \in \mathrm{v}_{j i} \\
\frac{\rho\left(v_{i}, v_{j i}\right)}{\rho\left(v_{i}, v_{p i}\right)-\rho\left(v_{i}, v_{j i}\right)} & \mathrm{v}_{i} \notin \mathrm{v}_{j i}\end{cases} \\
& \rho\left(v_{i}, v_{j i}\right)=\left|v_{i}-\frac{a_{j i}+b_{j i}}{2}\right|-\frac{b_{j i}-a_{j i}}{2} \\
& \left.\left|v_{j i}\right|=\left|b_{j i}-a_{j i}\right| ; \rho\left(v_{i}, v_{p i}\right)=\left|v_{i}-\frac{a_{p i}+b_{p i}}{2}\right|-\frac{b_{p i}-a_{p i}}{2}\right\}
\end{aligned}
$$

In the formula, $k_{j}\left(v_{i}\right)$ stands for the correlations about each evaluated factor index $f_{i}$ and $N_{j}$

\subsection{Calculating the weight about the Variable weights of the evaluated factors of the supply chain risk}

Weight coefficient is the quantified coefficient which be reflected about the importance of evaluated factor index of the supply chain risk, and its rationality played a pivotal role in identifying the risk category, this paper will get the weight coefficient by variable weights theory. To identify the weight coefficient rationally, different results will be got by different weight coefficients.

(1) Variable weights principle

Professor Wang Peizhuang put forward the variable-weight thought firstly, professor Li Hongxing gave the axiomatic definition about the variable-weights vector and the state variable-weights vector based on the factor space theory, and gave the model of the variable-weights synthesis and got the following important conclusions: hypothesize $X=\left(x_{1}, x_{2}, x_{3}, \ldots x_{n}\right)$ as the factor state vector, $W=\left(w_{1}, w_{2}, w_{3}, \ldots w_{n}\right)$ as factor constant-weighs vector, $S(X)=\left(S_{1}(X), S_{2}(X), S_{3}(X), \ldots, S_{n}(X)\right) \quad$ as stage 
variable-weights vector, so the variable-weights vector $W(X)=\left(w_{1}(X), w_{2}(X), w_{3}(X), \ldots, w_{n}(X)\right)$ can stands for the hadamard product of the unit of $W$ and $S(X)$, that is :

$$
w_{i}(X)=\frac{w_{i} S_{\mathrm{i}}(X)}{\sum_{k=1}^{n} w_{k} S_{k}(X)}, i=1,2, \ldots, n
$$

Professor Li-Hongxing researched the factor space theory systematically, and divided the stage variable-weights vector into three categories (punishment-based, incentive-based and hybrid), and that's due to the different role its played in the variable-weights synthesis. There is a stage variable-weights vector be constructed by $S_{i}\left(x_{1}, x_{2}, x_{3}, \ldots x_{n}\right)=e^{\alpha\left(x_{i}-\bar{x}\right)},(i=1,2,3 \ldots n)$ in the reference [8]. It is obviously to see that when $\alpha<0$, then formed the punishment-based stage variable-weights vector, when $\alpha>0$, then formed the incentive-based stage variable-weights vector, in the decision making process, when $\alpha$ is a negative number, that indicates each of the factors has a certain degree of balance requirements, and the smaller the number about $\alpha$, the higher the express request of the comprehensiveness. When $\alpha$ is a positive number, that indicates each of the factors does not has a certain degree of balance requirements, and the bigger the number is, the more attention will be take to the some factors which has bigger state value, if $\alpha=0$, then the variable-weights model turns to be the constant-weighs model.

(2) The weight $w_{j i}$ about the risk category of the to be evaluated Matter-element

$$
\begin{aligned}
& w_{j i}\left(k_{j}(v)\right)=\frac{w_{j i} S_{\mathrm{i}}\left(k_{j}(v)\right)}{\sum_{k=1}^{n} w_{j k} S_{k}\left(k_{j}(v)\right)}, i=1,2, \ldots, n \\
& S_{\mathrm{i}}\left(k_{j}(v)\right)=e^{\alpha\left(k_{j}\left(v_{i}\right) \overline{k_{j}(v)}\right)}
\end{aligned}
$$

\subsection{Determining the correlation about the to be evaluated} matter-element and the risk category $j$

The correlation about the to be evaluated matter-element and the risk category $j$ is:

$$
k_{j}(T)=\sum_{i=1}^{n} w_{j i} k_{j}\left(v_{i}\right)
$$

In the formula, $w_{j i}$ stands for the weight coefficient of the variable weight about each evaluated factor, and $\sum_{i=1}^{n} w_{j i}=1$

\subsection{Determining which risk category does the to be evaluated} matter-element belongs to

$$
\text { If } k_{j_{0}}(T)=\max \left(k_{j}(T)\right) \text {, then the to be evaluated }
$$

matter-element belongs to risk category ${ }^{j_{0}}$.or make

$$
\bar{k}_{j}(T)=\frac{k_{j}(T)-\min k_{j}(T)}{\max k_{j}(T)-\min k_{j}(T)} ; \quad \mathrm{j}^{*}=\frac{\sum_{j=1}^{m} j \bar{k}_{j}(T)}{\sum_{j=1}^{m} \bar{k}_{j}(T)}
$$

Then $\mathrm{j}^{*}$ is called as the variable eigenvalue of the risk category. For example: $j_{0}=2$, but $\mathrm{j}^{*}=2.3$, then that indicates its belongs to category 2 and partial to category 3(it's strictly to say its belongs to category 2,3 ), we can know the extent of partial to another category about the to be evaluated

$$
\text { matter-element from the value about } \mathrm{j}^{*}
$$

\section{Case study}

When evaluating the supply chain risk, we should determine the risk factors index system of supply chain and constant weights firstly, Then determine the extent or the category of the supply chain risk, Now using the variable weights extension matter-element model, to evaluate the risk of the supply chain of HengRui Pharmaceutical Co., Ltd (Listed Company) as core enterprise (HengRui supply chain for short), based on supply chain risk factor evaluation system proposed by the author.

\subsection{Setting up the supply chain risk factor evaluation system}

Based on the design principles, such as scientificity、 comparability、 operability and so on., and referring to some related date, combining some advices from experts and entrepreneurs, then setting up a supply chain risk factor evaluation system systematically and reasonably(refer to sheet 1), and calculating the weight of the constant weights based on the developed analytical hierarchical process(refer to reference [11])

$$
\begin{aligned}
W & =\left(w_{1}, w_{2}, \cdots ; w_{10}\right) \\
& =(0.0947,0.932,0.1531,0.0466,0.0846,0.1624,0.1535,0.1013,0.910,0.0196)
\end{aligned}
$$

\subsection{Using the variable weights extension Mmatter element} model to evaluate the hengRui supply chain

(1) Calculating the correlation about each risk category of the risk evaluated factor of the HengRui supply chain.

The author did the questionnaire survey, invited 10 experts to analysis and determine each evaluated factor index objectively, and refer to some relevant standards, then got the classical domain $\left\langle a_{j i}, b_{j i}\right\rangle, j=1,2,3,4,5$ and section domain $\left\langle a_{p i}, b_{p i}\right\rangle$ of the supply chain risk evaluated factor index by Comprehensive analysis. The author has organized 10 experts (college scholars and business managers) to score based on the score grading standards and fill in the expert evaluation score sheets, which about the evaluated factor indicator $f_{i}$ of the Hengrui supply chain risk, then got the evaluated matter-element matrix; calculated the correlation about each risk category of each evaluated factor indicator of the Hengrui supply chain, the results shown in table 1. 
Table 1 The relevance of the risk categories and the weight of variable weights in supply chain system of risk evaluated factors

\begin{tabular}{|c|c|c|c|c|c|c|c|c|c|c|}
\hline \multirow{2}{*}{$\begin{array}{l}\text { Supply chain system of risk evaluation } \\
\text { factors }\end{array}$} & \multicolumn{5}{|c|}{ Correlation } & \multicolumn{5}{|c|}{ The weight of the variable weights } \\
\hline & I & II & III & IV & $\mathrm{V}$ & I & II & III & IV & V \\
\hline $\begin{array}{l}\text { Raw material supply and the accident of product } \\
\text { operations } f_{1}\end{array}$ & -0.5000 & 0.0000 & 0.0000 & -0.5000 & -0.6667 & 0.0912 & 0.1708 & 0.1023 & 0.0365 & 0.0342 \\
\hline $\begin{array}{l}\text { Interrupted and delay in distribution and } \\
\text { transportation process } f_{2}\end{array}$ & -0.5000 & -0.3333 & 0.0000 & 0.0000 & -0.3333 & 0.0897 & 0.0863 & 0.1007 & 0.0976 & 0.0655 \\
\hline $\begin{array}{l}\text { Interrupted and delay in delivery scheduling and } \\
\text { service } f_{3}\end{array}$ & -0.5000 & -0.2500 & 0.5000 & -0 & -0.5000 & 0.1474 & 0.1675 & 0.4496 & 0.0973 & .0771 \\
\hline $\begin{array}{l}\text { Interrupted and delay in the market demand and } \\
\text { prediction } f_{4}\end{array}$ & -0.5000 & 0.0000 & 0.0000 & -0.5000 & -0.6667 & 0.0449 & 0.0840 & 0.0503 & 0.0180 & 0.0168 \\
\hline $\begin{array}{l}\text { The fair of the profit distribution and the } \\
\text { possibility of the leakage of core } \\
\text { competencies } f_{5}\end{array}$ & -0.5000 & -0.3333 & 0.0000 & 0.0000 & 33 & 0.0814 & 0.0783 & 0.0914 & 0.0886 & 0.0594 \\
\hline $\begin{array}{l}\text { Interrupted and delay in the information-sharing } \\
\text { process } f_{6}\end{array}$ & -0.7500 & -0.6667 & -0.5000 & 0.0000 & 00 & 0948 & 0.0772 & 0.0645 & 0.1701 & 0.2223 \\
\hline $\begin{array}{l}\text { The degree of the technical development, such as } \\
\text { stock, information and so on } f_{7}\end{array}$ & -0.6250 & -0.5000 & -0.2500 & 0.5000 & -0.2500 & 0.1151 & 0.1018 & 0.1006 & 0.4371 & 0.1274 \\
\hline $\begin{array}{l}\text { The degree of cultural conflict about the system of } \\
\text { node members, cultural and so on } f_{8}\end{array}$ & -0.8750 & -0.8333 & -0.7500 & -0.5000 & 0.5000 & 0.0461 & 0.0345 & 0.0244 & 0.0390 & 0.3769 \\
\hline $\begin{array}{l}\text { The leadership ability of the core enterprises and } \\
\text { the cooperation ability of non-core enterprises } f_{9}\end{array}$ & 0.0000 & 0.0000 & -1.0000 & -1.0000 & -1.0000 & 0.2381 & 0.1641 & 0.0133 & 0.0129 & 0.0169 \\
\hline $\begin{array}{l}\text { the degree of environmental instability about } \\
\text { natural, political, social and so on } f_{10}\end{array}$ & 0.0000 & 0.0000 & -1.0000 & -1.0000 & -1.0000 & 0.0513 & 0.0353 & 0.0029 & 0.0028 & 0.0036 \\
\hline
\end{tabular}

(2) Using formula (8) to calculate the weight of the variable weights.

(3) Determining the extent and the category about the risk category $j$ of the Hengrui supply chain based on the formula $(9) 、(10)$.

$$
\begin{aligned}
& \quad k_{1}(T)=-0.4107 ; \quad k_{2}(T)=-0.2279 ; \\
& \qquad k_{3}(T)=0.1329 ; \quad k_{4}(T)=0.1318 ; \quad k_{5}(T)=0.0219 \\
& \text { because of } \quad k_{j_{0}}(T)=\max \left(k_{j}(T)\right)=0.1329, \text { that }
\end{aligned}
$$

is $j_{0}=3$, so the risk category of Hengrui supply chain is moderate, the conclusion is coincide with the actual risk about Hengrui supply chain. in order to describe the risk category about Hengrui supply chain accurately, $j^{*}=6.4396$ can be calculated by formula(10), from $\mathrm{j}^{*}$, we can see that Hengrui supply chain risk is partial to a higher risk category, so it's reasonable to evaluate by the variable weights extension matter-element model.

\section{Concluding remarks}

Risk evaluation of the supply chain is an important content in risk management of supply chain. This paper set up a supply chain risk evaluation model based on variable-weights extenics matter-element, because of the complexity, uncertainty, and the characteristics of the supply chain risk. The characteristics of the model are: it can be decided according to local conditions to evaluate the quantity and variety of factors. It is easy with a good operability;

using a combination of qualitative and quantitative way, to avoid the random evaluated purely subjective and sensory in the past; using variable-weights theory to calculate the supply chain risk evaluated factors, make the weight of variable-weights reflect the importance of the evaluated factors reasonably; the credibility of evaluation results is very high, and also with very good practical value.

The paper also verified variable-weights based on matter-element extenics of supply chain risk evaluation model,

and have good scientificity and the effectiveness of the examples. Through evaluate the risk of supply chain, we not only can provide decision-makers with the total risk of the current situation, make them understand the stability of the supply chain, and enterprises can also be given the most intuitive reference to join in the supply chain.

\section{References}

[1]Prater E,Biehl M,Smith M A.International supply chain agility ,tradeoffs between flexibility and uncertainy[J].International Journal of Operational and Production Management,2001,21(5/6):823-829.

[2]Jukka H,Iris K,Urho P,etal.Risk management processes in supplier network[J].International Journal of Production Economics,2004,90:47-58.

[3]Ding Weidong,Liu Kai.Study on Risk of Supply Chain [J].China Safety Science Journal,2003,13(4):64-66.

[4] Xiao Mei-dan,Li Cong-dong.Assessment of Supply Chain Risk Based on Uncertainty Theory and Fuzzy Method[J].soft science,2007,21(5):27-30.

[5] Zhang Yan-ru; Chen Jing-xian.Fuzzy Assessment Method Research of Supply Chain Uncertain Risk Based on Predilection[J].Operations Research and Management Science, 2008,17 (1):7-12.

[6] Zhang Yi-bin; Chen Jun-fang.A Framework of Identifying Supply Chain Risks and Their Flexible Mitigating Polices [J].Industrial Engineering and Management,2008,(1):74-80.

[7] Li De-qing, Li Hong-xing. The properties and construction of state variable weight vectors [J]. Journal of Beijing Normal University (natural sciences version), 2002, 38 (4): 455-461.

[8]Li De-qing, Cui Hong-mei. Multifactor decision making based on hierarchical variable weights [J]. Journal of Systems Engineering, 2004, 19(3): 258-263.

[9]Li De-qing, Gu Yun-dong. Results on axiomatic definition of state variable weight vector [J].The Systems Engineering Theory and Practice, 2004, 24(5):97-102.

[10]Xie Kai-gui, Zhou Jia-qi. Research on combination forecasting model with time-variant weights $[\mathrm{J}]$. The Systems Engineering Theory and Practice, 2000, 20(7):36-40.

[11]Duan Shu-qiao. A method of variable weight synthetic evaluation for safety management of power business [J]. The Practice and Understanding of Mathematics, 2003, 33(8):17-23. 